\title{
Multiple and independent origins of short seeded alleles of $G S 3$ in rice
}

\author{
Noriko Takano-Kai1), Hui Jiang ${ }^{2,3)}$, Adrian Powell'), Susan McCouch ${ }^{2)}$, Itsuro Takamure ${ }^{4)}$, Naruto Furuya ${ }^{1)}$, \\ Kazuyuki Doi ${ }^{1,5)}$ and Atsushi Yoshimura*1) \\ 1) Faculty of Agriculture, Graduate School, Kyushu University, 6-10-1, Hakozaki, Higashi, Fukuoka 812-8581, Japan \\ 2) Department of Plant Breeding and Genetics, Cornell University, Ithaca, NY 14853, USA \\ 3) Present address: Donald Danforth Plant Science Center, 975 North Warson Road, St. Louis, Missouri 63132, USA \\ 4) Graduate School of Agriculture, Hokkaido University, Kita9-Nishi9, Kita, Sapporo, Hokkaido 060-8589, Japan \\ 5) Present address: Graduate School of Bioagricultural Sciences, Nagoya University, Chikusa, Nagoya 461-8601, Japan
}

GRAIN SIZE 3 (GS3) is a cloned gene that is related to seed length. Here we report the discovery of new deletion alleles at the GS3 locus, each of which confer short seed. We selected ten short seeded cultivars from a collection of 282 diverse cultivars. Sequence analysis across the GS3 gene in these ten cultivars identified three novel alleles and a known allele that contain several independent deletion(s) in the fifth exon of GS3. These independent deletion variants each resulted in a frameshift mutation that caused a premature stop codon, and they were functionally similar to one another. Each coded for a truncated gene product that behaved as an incomplete dominant allele and conferred a short seeded phenotype. Haplotype analysis of these sequence variants indicated that two of the variants were of japonica origin, and two were from indica. Transformation experiments demonstrated that one of the deletion alleles of GS3 decrease the cell number in the upper epidermis of the glume, resulting in a significant reduction in seed length. The multiple and independent origins of these short seeded alleles indicate that farmers and early breeders imposed artificial selection favoring short seeds.

Key Words: seed length, frameshift mutation, haplotype analysis, diversity, cell number.

\section{Introduction}

Rice (Oryza sativa L.) is the most important staple crop for billions of people worldwide. Given rapid population and income growth in many rice-eating parts of the world, the breeding of high-yielding and high-quality rice cultivars is critical to meet growing demand (Khush 1999). Rice seed shape is a breeding target because it directly affects grain weight, which is an important component of grain yield. In addition, rice seed shape is an important determinant of consumer preference around the world. Long, slender grains are preferred by many consumers in India, Pakistan, Thailand, China and the United States, while consumers in Japan, South Korea and Sri Lanka prefer short, bold grained varieties (Juliano and Villareal 1993, Unnevehr et al. 1992).

There is greater variation for seed length found among cultivated varieties than in wild rice, probably due to human selection (Takano-Kai et al. 2009). Preferences for different seed sizes and shapes are dependent on how rice is cooked, processed and consumed. For example, increasing the amount of rice bran available for extraction of rice oil can be most easily accomplished by reducing the size of the rice

Communicated by T. Sasaki

Received August 29, 2012. Accepted November 1, 2012.

*Corresponding author (e-mail: ayoshi@agr.kyushu-u.ac.jp) seed, which increases the surface area per volume of brown rice. There is evidence that breeders have selected for short seed size as well as large seed size in rice (Mikami et al. 2004).

Many genes are known to control seed size and several independent studies based on inter- and intraspecific crosses of rice have previously identified quantitative trait loci (QTLs) associated with seed length (Li et al. 2004, Redoña and Mackill 1998, Tan et al. 2000, Tsunematsu et al. 1995), some of which have been identified and characterized. GRAIN SIZE3 (GS3) encodes a protein with several conserved domains, including a phosphatidylethanolaminebinding protein (PEBP)-like domain, a transmembrane region (TM), a putative tumour necrosis factor receptor/nerve growth factor receptor (TNFR/NGFR) family domain and a von Willebrand factor type C (VWFC) domain (Fan et al. 2006, Takano-Kai et al. 2009). GRAIN WEIGHT2 (GW2) encodes an unknown RING-type protein with E3 ubiquitin ligase activity (Song et al. 2007). The identical gene of $q S W 5$ and GW5 has no apparent homolog in the database but was shown to interact with the polyubiquitin-proteasome pathway to regulate cell division during seed development (Shomura et al. 2008, Weng et al. 2008).

Among the genes known to regulate seed length, GS3 is an interesting case because its mutants both positively and negatively regulate seed length. GS3 consists of five exons. 
The wild type allele of GS3 results in a medium seed phenotype. A C-to-A nonsense mutation in the second exon of GS3 results in a long seeded phenotype (Fan et al. 2006, Takano-Kai et al. 2009). A 1-bp deletion in the fifth exon of $G S 3$, leading to a frameshift mutation at the $\mathrm{C}$ terminus, produced a short seeded phenotype due to a truncated protein that lacked the TNFR/NGFR and VWFC domains (Mao et al. 2010).

Here we report the identification of novel, incomplete dominant alleles at GS3 that all confer short seeds. The variants were identified based on sequencing across the GS3 locus in ten short seeded cultivars, each having seed length $<6.5 \mathrm{~mm}$. These alleles represent similar functional mutations, suggesting that the fifth exon of the GS3 gene is a hotspot for mutation and has been the target of selection by many independent groups of humans during the evolution of $O$. sativa. We also examined sequence haplotypes across the $G S 3$ gene in short seeded cultivars to identify the subpopulation origin of these alleles.

\section{Materials and Methods}

Plant materials used in survey for short seeded cultivars

We surveyed seed size in 281 diverse cultivars maintained in the Plant Breeding Laboratory, Faculty of Agriculture, Kyushu University (Supplemental Table 1). Eighty one of these cultivars overlapped with the 235 cultivars of $O$. sativa previously investigated for grain size (Takano-Kai et al. 2009). Included in this collection was the short seeded rice mutant line, H343, maintained in Hokkaido University. H343 carries the MINUTE (Mi) locus for short seeds that was previously mapped near GS3 on chromosome 3 (Fraker et al. 2004, Takamure et al. 1991, Takeda and Saito 1977). Seed length in the 282 rice strains was measured using a caliper (KORI Dial Caliper, KORI SEIKI, Tokyo, Japan).

\section{Sequence analysis of $G S 3$}

Approximately $6 \mathrm{kbp}$ of genomic sequence across the $G S 3$ gene was generated from the ten short grained strains. This region contained 1122-bp upstream from the start codon and 119-bp downstream from the stop codon. Three pairs of PCR primers were designed to amplify overlapping regions within the gene, and internal primers within each amplicon were designed to sequence the PCR products. PCR reactions to generate the sequencing template were performed in $25 \mu \mathrm{l}$ of reaction mixture containing 1x KOD-

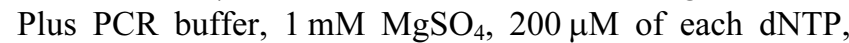
$0.2 \mu \mathrm{M}$ of each primer, 1 unit of KOD-Plus DNA polymerase (TOYOBO, Osaka, Japan) and approximately $25 \mathrm{ng}$ of template DNA in a GeneAmp PCR 9700 system (Applied Biosystems, Foster City, CA, USA). The PCR program used was $95^{\circ} \mathrm{C}$ for $2 \mathrm{~min}$, followed by 35 cycles of $98^{\circ} \mathrm{C}$ for $30 \mathrm{~s}$, $60^{\circ} \mathrm{C}$ for $30 \mathrm{~s}$ and $68^{\circ} \mathrm{C}$ for $5 \mathrm{~min}$. The PCR products were sequenced with the BigDye Terminator v3.1 cycle sequencing kit (Applied Biosystems, Foster City, CA, USA) using the ABI 3130X genetic analyzer. Sequences were assembled and aligned using the Sequencher program (Gene Codes, Ann Arbor, MI).

\section{Plant materials for genetic analysis}

A medium seeded temperate japonica cultivar, Taichung 65 (T65), a short seeded aromatic cultivar, JC73-4, an $\mathrm{F}_{1}$ and an $\mathrm{F}_{2}$ population derived from a cross between $\mathrm{T} 65$ and JC73-4 were genetically analyzed. The seed lengths of the T65, JC73-4, $\mathrm{F}_{1}$ and $\mathrm{F}_{2}$ population were measured by the method described above.

\section{DNA extraction and genetic analysis}

Genomic DNA for the simple sequence repeat (SSR) analysis was extracted from freeze-dried leaf samples using a modified potassium acetate-SDS method (Dellaporta et al. 1983). The PCR reactions were performed in $15 \mu \mathrm{l}$ of reaction mixture containing $50 \mathrm{mM} \mathrm{KC1}, 10 \mathrm{mM}$ Tris- $\mathrm{HCl}$ (pH 9.0), $1.5 \mathrm{mM} \mathrm{MgCl} 2,200 \mu \mathrm{l}$ of each dNTP, $0.2 \mu \mathrm{M}$ of each primer, 0.75 units Taq polymerase (TaKaRa, Otsu, Japan) and approximately $25 \mathrm{ng}$ template DNA in a GeneAmp PCR 9700 system (Applied Biosystems, Foster City, CA, USA). The PCR program used was $95^{\circ} \mathrm{C}$ for $5 \mathrm{~min}$, followed by 35 cycles of $95^{\circ} \mathrm{C}$ for $30 \mathrm{~s}, 55^{\circ} \mathrm{C}$ for $30 \mathrm{~s}$ and $72^{\circ} \mathrm{C}$ for $30 \mathrm{~s}$. PCR products were run in $4 \%$ agarose gels (Agarose HT; Amresco Inc., Solon, OH, USA) in 0.5x TBE buffer.

\section{Vector construction and plant transformation}

To generate the GS3 construct used to introduce the 320bp deletion into the medium seeded cultivar, Nipponbare, a construct used previously by Takano-Kai et al. (2009) was used as the mutagenesis template. The primers used for the mutagenesis were GCAGCAGCAGCTCGGCCTCTTGA GGTTGAA and GCGCGTACTTAATTAGCTCAAGATT AGTTCTCGATGATGATC, each containing a modified phosphoric acid at the $5^{\prime}$ end. PCR reactions for mutagenesis were performed in $50 \mu \mathrm{l}$ of reaction mixture containing $1 \mathrm{x}$

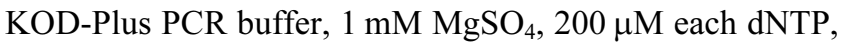
$0.2 \mu \mathrm{M}$ each primer, 1 unit of KOD-Plus DNA polymerase and approximately $1 \mu \mathrm{g}$ template construct in a GeneAmp PCR 9700 system. The PCR program used was $95^{\circ} \mathrm{C}$ for $2 \mathrm{~min}$, followed by 20 cycles of $98^{\circ} \mathrm{C}$ for $10 \mathrm{~s}$ and $68^{\circ} \mathrm{C}$ for $17 \mathrm{~min}$. The PCR product was re-circularized using a DNA ligation kit, Ligation High (TOYOBO, Osaka, Japan) and was introduced into competent bacteria Escherichia coli DH5 $\alpha$ (TOYOBO). Positive colonies were identified by PCR screening using the two primer sets TCCCACAAAA CCATCAACTTGTTA and CAAGCAGAGCAAGGCAAT CAA and GGGAATAGGGATTCCACACG and AATGG TGATGCCCAGCTTTT. The presence of the 320-bp deletion was confirmed by DNA sequencing. This construct (referred to here as the GS3-d construct) (Supplemental Fig. 1) was introduced into Nipponbare by Agrobacteriummediated transformation (Toki 1997).

GS3 from Nipponbare was used as a control (referred to here as the GS3 construct) (Supplemental Fig. 1). In 
addition, the RNA interference (RNAi) trigger region was amplified by PCR with the primers CACCCGCGAGATC GGATTCCTC and AGCGACACGGACTCTTCGTT and then inserted into the $\mathrm{pENTR/D-TOPO}$ cloning vector (Invitrogen, Carlsbad, CA, USA) to yield an entry vector. The RNA silencing vector was produced by an LR Clonasecatalysed reaction (Invitrogen) between the entry vector and pANDA (Miki and Shimamoto 2004, referred to here as the GS3-p construct). The GS3-p construct (Supplemental Fig. 1) was also used as a control. These two constructs, GS3 and GS3-p, were also introduced into Nipponbare by Agrobacterium-mediated transformation.

For measurement of seed length of the transformants, five seeds from each of five $\mathrm{T}_{0}$ plants were used.

Measurement of cell number and cell size in the upper epidermis of the rice glume

Mature seeds were coated with gold-palladium and viewed under a scanning electron microscope (SEM; JSM5200, Jeol, Tokyo, Japan). Longitudinal surface images of each seed were recorded as sequential JPEG files. The sequential images of each seed were then merged into a single image using Photoshop software. Longitudinal rows of tubercles were observed on the outer surface of the rice hull. These tubercles are formed by accumulation of silicic acid in the cell walls of the upper epidermal cell (Takeoka 1976). The cell number of upper epidermis in the longitudinal tubercles was counted from the apex to the base of the seed in transgenic plants. The cell number of upper epidermal cells of each transgenic line was calculated from the mean of three values from each of five plants. In addition, the length of upper epidermal cells of the transgenic plants was measured by determining the length between the tubercles in the middle of the glume. The cell size of upper epidermal cells of the transgenic lines was calculated from the mean of 120 values per seed from five independent seeds. The number and length of cells in the palea and lemma were obtained using the ImageJ software (http://rsb.info.nih.gov/ij/).

\section{Results}

Sequence analysis of GS3 in short seeded cultivars

A diverse collection of 282 strains of $O$. sativa (Supplemental Table 1) was evaluated for seed size. The seed length of the 282 strains varied from $4.99 \mathrm{~mm}$ to $10.52 \mathrm{~mm}$. In our previous study, we surveyed the sequence variant of the GS3 locus among 54 rice strains and no functional nucleotide polymorphism was found except for a nonsense mutation in the second exon, which results in a long seeded phenotype (Takano-Kai et al. 2009). All of the 54 strains showed the seed length of $6.56-10.67 \mathrm{~mm}$. Therefore, ten out of 282 strains $(3.5 \%)$ that had a seed length $<6.5 \mathrm{~mm}$ (Fig. 1A and Table 1) were subsequently subjected for sequence analysis of $G S 3$

A total of 78 SNPs and 26 indels relative to Nipponbare were identified among the selected 10 strains in the se- quence analysis of $G S 3$. Of these mutations, only deletion(s) in the fifth exon of $G S 3$ were observed in all 10 strains. JC73-4, JC149, JC157, JC101 and ABRI showed a 320-bp deletion 5064-bp downstream from the start codon (referred to as the 5064-bp site); H343 and Tumo-Tumo had a 13-bp deletion at the 5000-bp site; ARC7291 and TAL214 had a 4bp deletion at the 5021-bp site (similar to the allele found in Chuan 7 by Mao et al. 2010); Podiwi A8 had a 1-bp deletion at the 4953-bp site and a 3-bp deletion at the 5016-bp site (1+3-bp deletions, Fig. 1B).

Deletion of the fifth exon alleles in GS3 causes a frameshift mutation resulting in a premature stop codon

The 320-bp, 13-bp, 4-bp and 1+3-bp deletions of GS3 each cause a frameshift that generates a premature stop codon (Fig. 2). The deduced amino acid sequence of the 320-bp deletion allele of GS3 (JC73-4, JC149, JC157, JC101 and ABRI) indicated that the product lacked a part of the TNFR/NGFR domain and the entire VWFC domain. The deduced amino acid sequence of the 13-bp deletion allele (H343 and Tumo-Tumo) indicated that the product lacked all of the TNFR/NGFR and VWFC domains. Although the three residues of the TM of the 13-bp deletion allele were changed, the domain prediction database (http://www.ebi. ac.uk/Tools/pfa/iprscan/) found that the product had a TM. The deduced amino acid sequence of the 4-bp deletion allele of GS3 (ARC7291 and TAL214) revealed that the product also lacked all of the TNFR/NGFR and VWFC domains, and these sequences were the same as that of Chuan 7 (Mao et al. 2010). The deduced amino acid sequence of the $1+3$-bp deletion allele of GS3 (Podiwi A8) also lacked the TM, three domains of TNFR/NGFR and VWFC, and encoded a different C-terminus compared to the GS3 locus of Nipponbare.

Mao et al. (2010) demonstrated that the functional shortseeded allele in the variety Chuan 7 encoded a gene product that lacked the TNFR/NGFR and VWFC domains. Therefore, since all of the GS3 variants found in this study produce GS3 products lacking the normal TNFR/NGFR and VWFC domains, we conclude that this is the reason they confer short seeded phenotypes.

\section{Origin of deletion alleles by haplotype analysis}

To investigate the origin of the deletions in the 5 th exon of $G S 3$, we examined $G S 3$ sequence haplotypes in the 10 short seeded varieties. A total of 104 SNPs/indels were identified in the 10 short seeded strains relative to Nipponbare. Sixty one of these SNPs/indels were identical to those used for haplotype analysis in a previous study (Takano-Kai et al. 2009) and they were used to determine the origin of the haplotypes found in the 10 short seeded cultivars in this study.

The GS3 gene haplotypes from the 10 short seeded cultivars were classified into three groups. The first gene haplotype group included cultivars carrying the 320-bp deletion (JC73-4, JC149, JC157, JC101 and ABRI) and those carrying the 13-bp deletion (H343 and Tumo-Tumo). This gene 


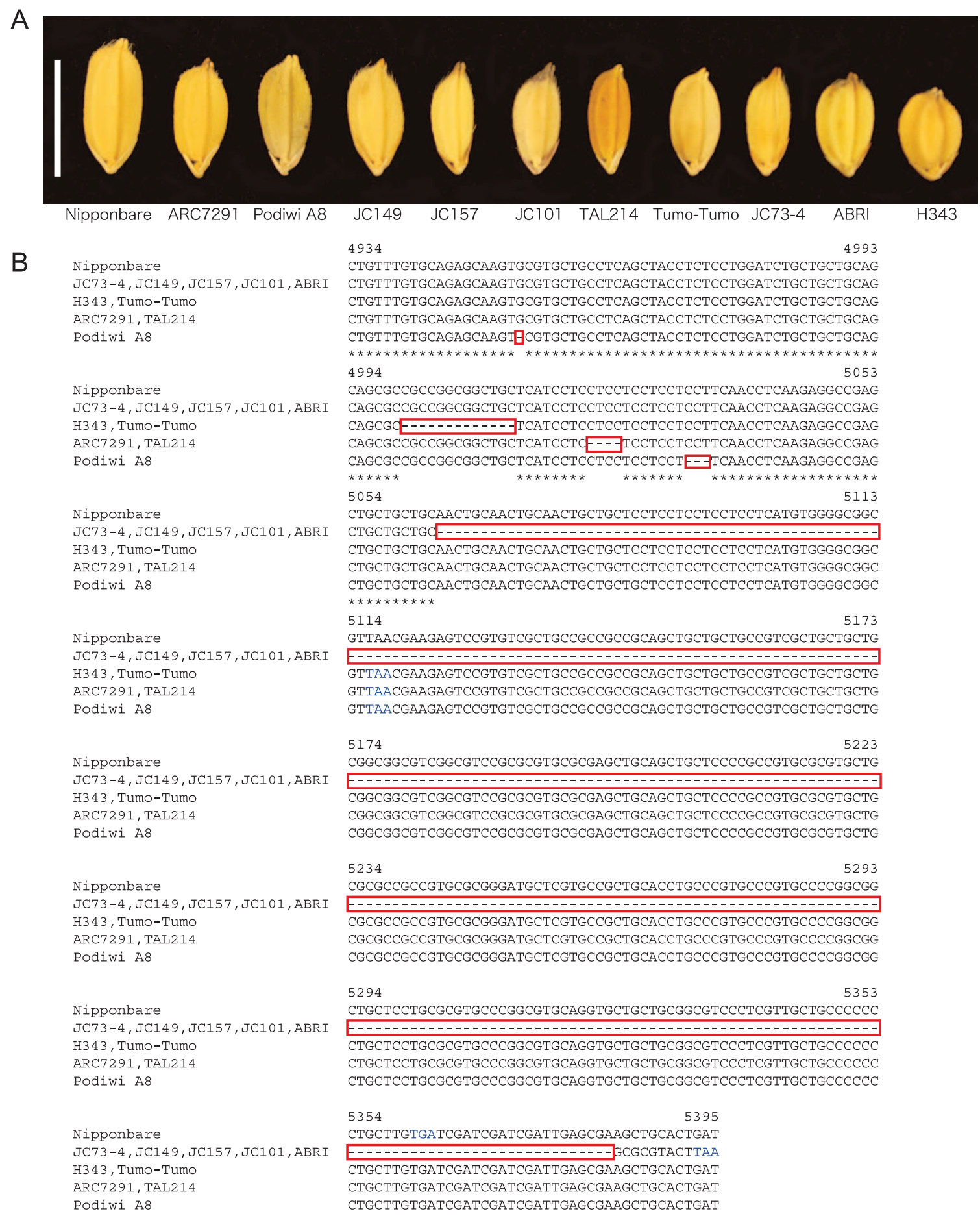

Fig. 1. Seed length comparison of the selected short seeded cultivars and sequence analysis of GS3 in the short seeded cultivars. (A) The seeds of Nipponbare and short seeded cultivars. From left to right, Nipponbare, ARC7291, Podiwi A8, JC149, JC157, JC101, TAL214, Tumo-Tumo, JC73-4, ABRI and H343. Bar is $6.5 \mathrm{~mm}$. (B) The nucleotide sequences from the fifth exon of Nipponbare GS3 are shown. The red boxes indicate the sites of deletion. Blue characters indicate the stop codon. The numbers indicate the nucleotide numbers of Nipponbare GS3.

haplotype group corresponded to the previously identified gene haplotype group 1 (GH1) that was reported to be of japonica origin (Takano-Kai et al. 2009). The second gene haplotype included cultivars carrying the 4-bp deletion (ARC7291 and TAL214) and corresponded to GH9 that was previously reported to be of indica origin. The last gene haplotype included the cultivar carrying the $1+3$ deletion (Podiwi A8) and was most similar to GH10, previously reported to be of indica origin (Fig 3). These results demonstrated that the 320-bp and 13-bp deletions occurred in a 
Table 1. Plant material used for the sequence analysis of GS3

\begin{tabular}{lcc}
\hline \hline Accession name & Origin of Country & Seed length $(\mathrm{mm} \pm$ SD) \\
\hline JC73-4 & India & $5.92 \pm 0.14$ \\
JC149 & India & $6.26 \pm 0.18$ \\
JC157 & India & $6.14 \pm 0.27$ \\
JC101 & India & $6.00 \pm 0.07$ \\
ABRI & Bhutan & $5.66 \pm 0.15$ \\
Tumo-Tumo & Malaysia & $5.94 \pm 0.18$ \\
ARC7291 & India & $6.46 \pm 0.05$ \\
TAL214 & Taiwan & $5.97 \pm 0.15$ \\
Podiwi A8 & Sri lanka & $6.43 \pm 0.16$ \\
H343 & Japan & $4.99 \pm 0.01$ \\
\hline
\end{tabular}

japonica-like ancestor and that the 4-bp and $1+3$-bp deletions occurred in an indica-like ancestor.

\section{Analysis of the JC73-4 with 320-bp deletion allele}

We performed genetic analysis of JC73-4 with the 320bp deletion allele. The $\mathrm{F}_{1}$ and $\mathrm{F}_{2}$ populations derived from a cross between T65 and JC73-4 were used. T65 with wildtype allele of $G S 3$ showed medium seed length and the $\mathrm{F}_{1}$ plants showed an intermediate seed length $(6.62 \pm 0.11 \mathrm{~mm})$ relative to the two parents (Fig. 4A). The seed length of the $\mathrm{F}_{2}$ population showed a trimodal frequency distribution (Fig. 4B). The 85 plants in the $\mathrm{F}_{2}$ population were divided into three groups: the short seed group (seed length: 5.41$5.93 \mathrm{~mm}$ ), the intermediate seed group (seed length: 6.01-
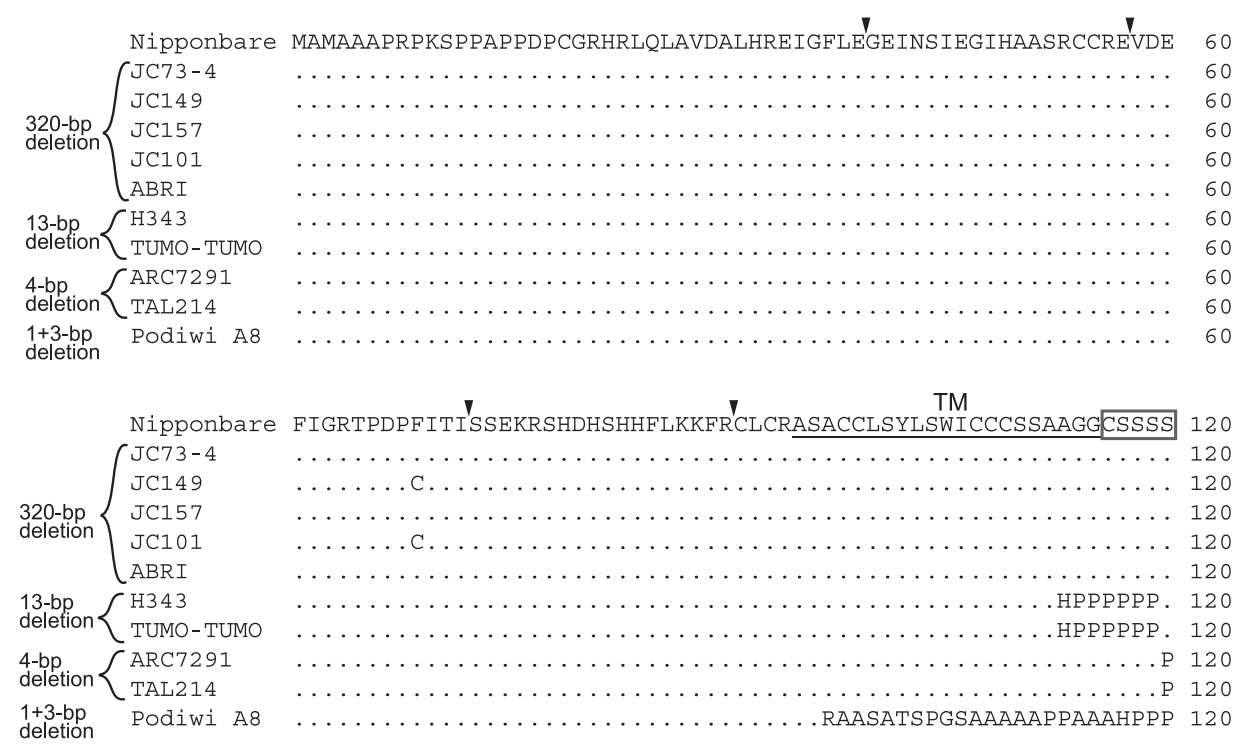

TNFR/NGFR

\begin{tabular}{|c|c|c|c|}
\hline & Nipponbare & SSSFNLKRPSCCCNCNCNCCSSSSSSCGAALTKSPCRCRRRSCCCRRCCCGGVGVRACAS & 180 \\
\hline & JC73-4 & $\ldots \ldots \ldots$ ART & 136 \\
\hline & JC149 & $\ldots \ldots \ldots \ldots$ ART & 136 \\
\hline $\begin{array}{l}320-b p \\
\text { deletion }\end{array}$ & JC157 & $\ldots \ldots \ldots \ldots$ ART & 136 \\
\hline & JC101 & $\ldots \ldots \ldots$ ART & 136 \\
\hline & LABRI & $\ldots \ldots \ldots \ldots$ ART & 136 \\
\hline 13-bp & $\mathrm{H} 343$ & - - - TSRG. AAAATATAT - - - - - - - . . PPPP. HVG . . & 46 \\
\hline deletion & TUMO-TUMO & --- TSRG . AAAATATAT $-\cdots-\cdots,-\cdots$ PPPP. HVG . . & 46 \\
\hline 4-bp. & ARC7291 & PP. TSRG. AAAATATAT $-\cdots-\cdots,-\ldots$ PPPP. HVG . . & 149 \\
\hline deletion & TAL2 14 & PP. TSRG. AAAATATAT $-\cdots$ & 149 \\
\hline $\begin{array}{l}\text { 1+3-bp } \\
\text { deletion }\end{array}$ & Podiwi A8 & PP. TSRG. AAAATATAT $\cdots \cdots$ & 149 \\
\hline & & 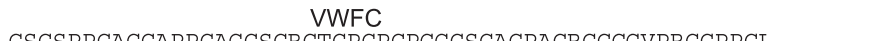 & \\
\hline & Nipponbare & CSCSPPCACCAPPCAGCSCRCTCPCPCPGGCSCACPACRCCCGVPRCCPPCL & 233 \\
\hline & ( JC73-4 & & 36 \\
\hline & JC149 & & 36 \\
\hline 320-bp & JC157 & & 136 \\
\hline deletion & JC101 & & 36 \\
\hline & ABRI & & 136 \\
\hline 13-bp & $\mathrm{H} 343$ & & 46 \\
\hline & TUMO-TUMO & & 146 \\
\hline 4-bp & ARC7291 & & 149 \\
\hline & TAL2 214 & & 149 \\
\hline $\begin{array}{l}1+3-b p \\
\text { deletion }\end{array}$ & Podiwi A8 & & 149 \\
\hline
\end{tabular}

Fig. 2. Alignment of the deduced amino acid sequence of $G S 3$ of Nipponbare and the 10 short seeded cultivars. The single solid underline indicates the transmembrane region (TM), the double underlines indicate the TNFR/NGFR family cysteine-rich domain (TNFR/NGFR) and the boxes indicate the von Willebrand factor type C (VWFC) domain. The triangles indicate the exon/exon boundaries of GS3. Dots indicate the same residues as Nipponbare sequence and hyphens indicate the deletions. Numbers at right side indicate the number of residues. 


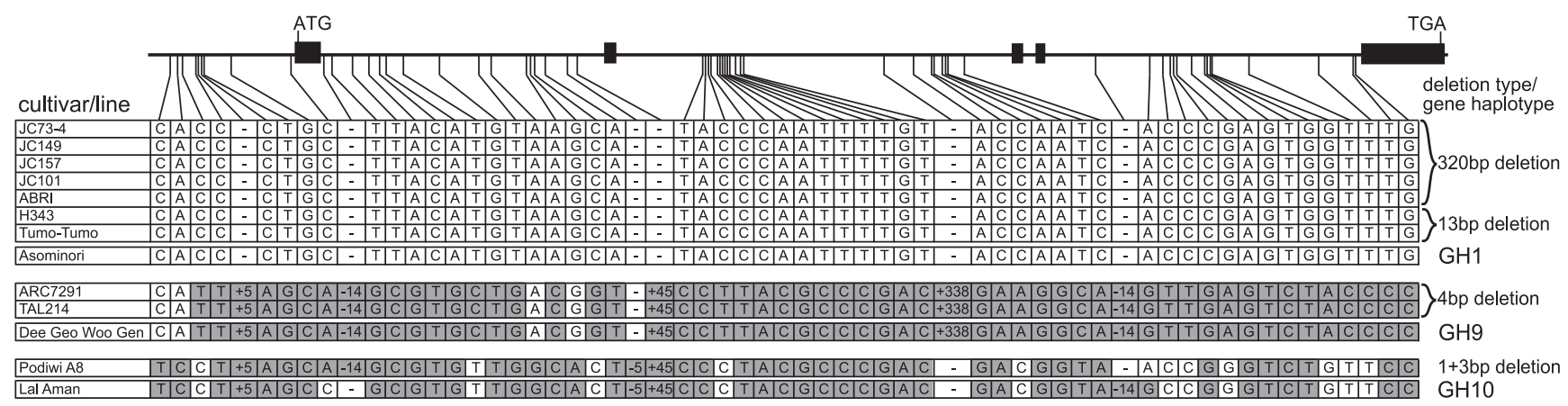

Fig. 3. Haplotype variation of $G S 3$ in short seeded cultivars. The gene model for $G S 3$ containing five exons and comprising approximately $6.2 \mathrm{~kb}$ is shown horizontally along the top. SNP positions with GS3 are connected by lines to the table below. Columns indicate the distribution of polymorphisms at each SNP/indel positions. Numbers indicate the size of an indel relative to Nipponbare. Asominori is one of cultivars belonging to japonica haplotype GH1, Dee Geo Woo Gen is one of cultivars belonging to indica haplotype GH9 and Lal Aman is a cultivar belonging to indica haplotype GH10 (Takano-Kai et al. 2009). White indicates the common GH1 and grey indicates a variant SNP allele.

$6.86 \mathrm{~mm}$ ) and the medium seed group (seed length: 6.92$7.77 \mathrm{~mm}$ ); 17 plants had short seeds, 43 plants had intermediate seeds and 25 plants had medium seeds. This was consistent with a $1: 2: 1$ segregation ratio (chi-square $=1.518$, $P=0.468$ ), indicating that short seed length was governed by a single, incompletely dominant allele coming from JC73-4. We designed PCR primers to detect the 320-bp deletion and genotyped each individual of the $\mathrm{F}_{2}$ population. All T65 homozygous plants had medium seeds, the heterozygous plants and three JC73-4 homozygotes had intermediate seeds and 17 of the JC73-4 homozygotes had short seeds (Fig. 4B). Thus, seed length co-segregated with the 320-bp deletion in the GS3 gene.

Next, we performed a set of transgenic experiments to obtain direct evidence that the 320-bp deletion of GS3 caused the short seeded phenotype. Using site-directed mutagenesis, a plasmid construct (GS3-d construct) was generated from the Nipponbare sequence containing the 320-bp deletion. Nipponbare GS3 (GS3 construct) was used as a control (Supplemental Fig. 1). Each construct was transfected into Nipponbare.

The seed length of the regenerated transgenic plants transformed with the GS3-d construct (GS3-d transformant, $6.43 \pm 0.12 \mathrm{~mm})$ was significantly shorter than that of the control (GS3 transformant, $7.12 \pm 0.14 \mathrm{~mm}$ ) (Fig. 4C). These results indicate that the 320-bp deletion allele is a functional form of GS3 that causes the short seeded phenotype. The culm length of GS3-d transformant was shorter than that of GS3-transformant (data not shown).

\section{GS3 decreases cell number in the upper epidermis of the} glume

To investigate the effect of GS3 on seeds at the cellular level, we measured the total number of cells and mean cell length in the upper epidermis of the glume using transgenic plants.

We measured the cell number and cell size in the upper epidermis of the lemma and palea of GS3 transformants,
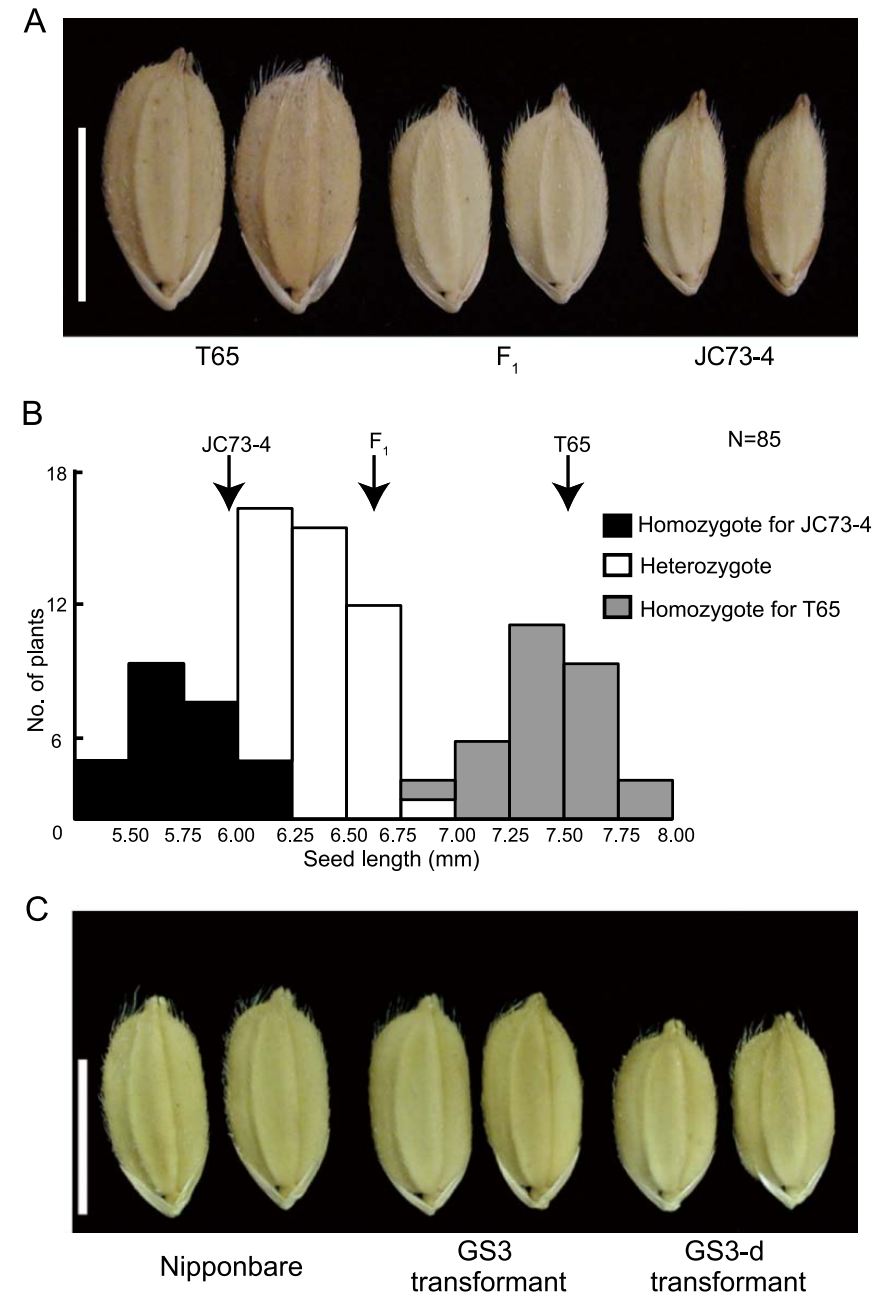

Fig. 4. Genetic analysis of seed length in JC73-4 and transformation experiment for $G S 3$ including the 320-bp deletion. (A) The seeds of T65, $\mathrm{F}_{1}$ and JC73-4. Bar is $5 \mathrm{~mm}$. (B) Frequency distribution of the seed length in the $\mathrm{F}_{2}$ population, classified by the genotypes of a marker to detect the 320-bp deletion. (C) The seeds of Nipponbare, GS3 transformants and GS3-d transformants. Bar is $5 \mathrm{~mm}$. 

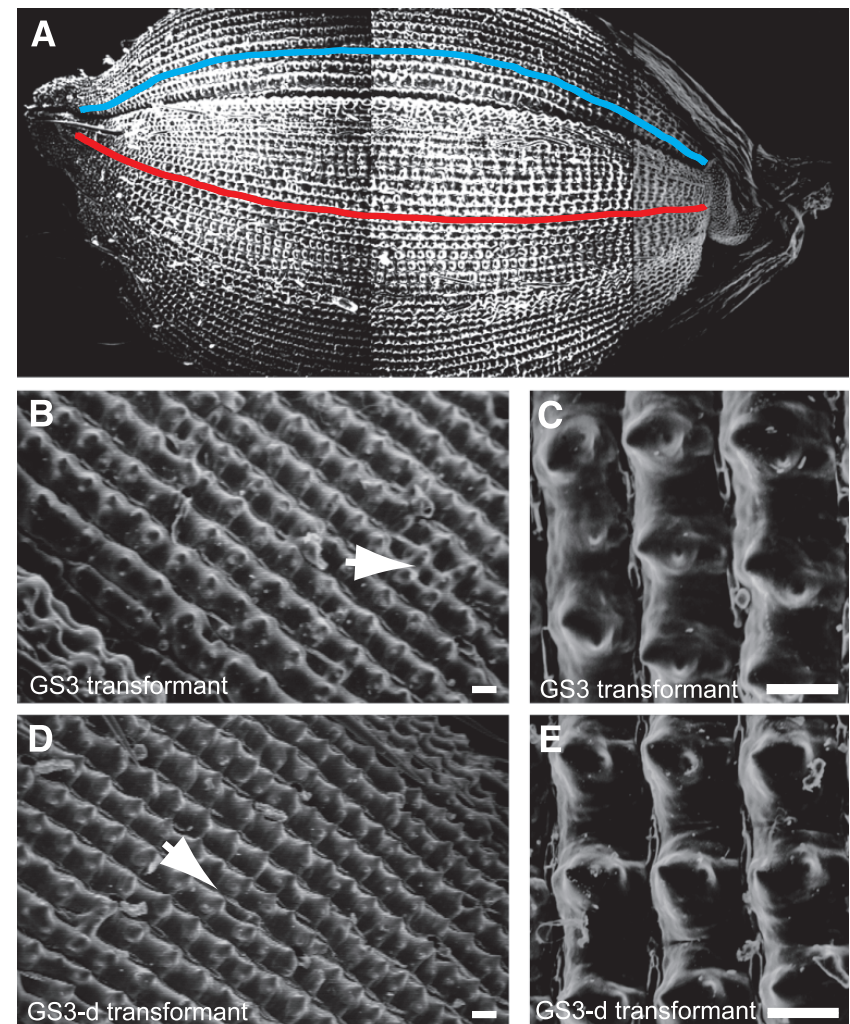

E
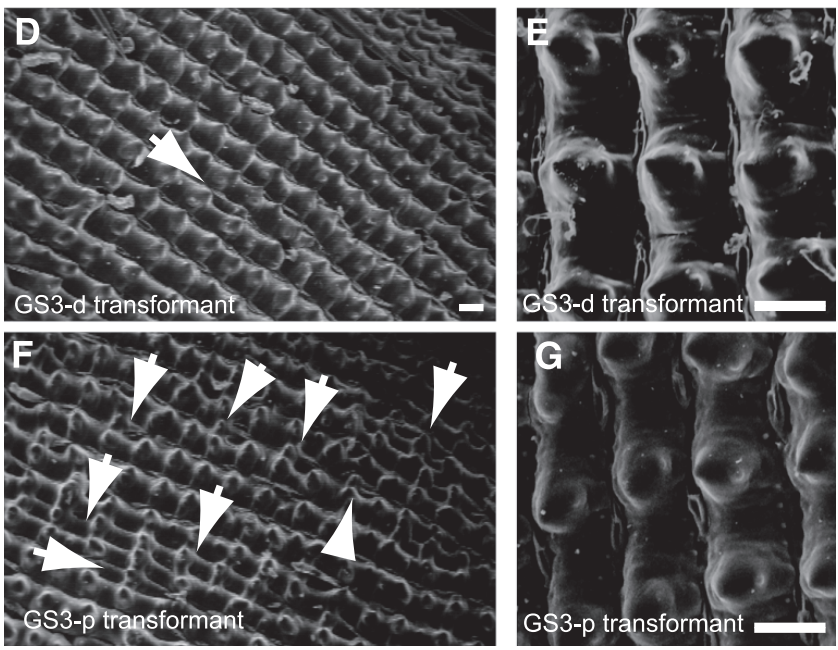

Fig. 5. Scanning electron micrographs of the upper epidermis in the transgenic plants. (A) The three sequential images of a seed were merged into a single image. The cell numbers of the upper epidermis were counted in the longitudinal tubercles that are formed from the apex to the base of a seed. Red and blue lines indicate a row of longitudinal tubercles at the lemma and palea, respectively. (B, C) The upper epidermis in the GS3 transformant. (D, E) The upper epidermis in the GS3-d transformant. (F, G) The upper epidermis in the GS3-p transformant. Arrows indicate the irregular thin rows. Bars are $50 \mu \mathrm{m}$.
GS3-d transformants and GS3-p transformants using SEM (Fig. 5). Although the cell size of the GS3-d transformants was smaller than that of the GS3 and GS3-p transformants, no significant difference was observed between the cell sizes of the GS3 and GS3-p transformants in the lemma or the palea. Conversely, significant differences were observed in the cell numbers of the GS3, GS3-d and GS3-p transformants in both the lemma and palea (Table 2); namely, the 320-bp deletion decreased the cell number, while a knockdown construct utilizing RNAi increased the cell number. These results indicate that GS3 controls cell number in the upper epidermis of the glume.

The surface of the outer glume of rice has rows of stripes along the longitudinal axis of the rice seed. The GS3-p transformant glume had more irregular, thinner rows of stripes than GS3 and GS3-d transformant glumes (Fig. 5). The loss of function of GS3 leads to an increase in irregular cells.

\section{Discussion}

GS3 has multiple alleles conferring the short seeded phenotype

In this study, we evaluated the seed size of 282 diverse rice strains and identified three novel GS3 alleles with independent deletions that all map to the fifth exon of the gene (320-bp, 13-bp and 1+3-bp deletion). We also identified a variety carrying a fourth deletion (4-bp deletion) that had been previously shown to be a determinant of short seed size in rice (Mao et al. 2010). Using genetic and transgenic analysis, we demonstrated that the 320-bp deletion causes a short seeded phenotype. The other two alleles discovered here (13-bp, 1 + 3-bp deletions) caused premature stops and were found to give rise to identical GS3 amino acid sequences, with gene products that were identical to those generated by the 4-bp deletion allele and 320-bp deletion allele found in Chuan 7 (Mao et al. 2010) and in JC73-4 (this study), respectively. From these results, we conclude that diverse deletion alleles of GS3 are all responsible for the short seeded phenotype.

The short seeded alleles of GS3 have both japonica and indica origins

We showed that the 320-bp and 13-bp deletions in the fifth exon of GS3 occurred in a japonica-like ancestor, and the 4-bp and 1+3-bp deletions occurred in an indica-like

Table 2. Cell number and size of the upper epidermis of transgenic lines

\begin{tabular}{lccccc}
\hline \hline \multirow{2}{*}{ Line } & \multicolumn{2}{c}{ Palea } & & \multicolumn{2}{c}{ Lemma } \\
\cline { 2 - 3 } \cline { 5 - 6 } & Cell number & Cell size $(\mu \mathrm{m} \pm \mathrm{SE})$ & & Cell number & Cell size $(\mu \mathrm{m} \pm \mathrm{SE})$ \\
\hline GS3 transformant & $92.17 \pm 1.93^{\mathrm{a}}$ & $79.71 \pm 1.98^{\mathrm{a}}$ & & $82.86 \pm 1.81^{\mathrm{a}}$ & $95.97 \pm 1.95^{\mathrm{a}}$ \\
GS3-d transformant & $72.33 \pm 1.85^{\mathrm{b}}$ & $66.66 \pm 1.33^{\mathrm{b}}$ & & $71.40 \pm 1.45^{\mathrm{b}}$ & $80.96 \pm 1.72^{\mathrm{b}}$ \\
GS3-p transformant & $109.00 \pm 1.14^{\mathrm{c}}$ & $84.30 \pm 1.41^{\mathrm{a}}$ & & $98.31 \pm 1.65^{\mathrm{c}}$ & $92.45 \pm 1.31^{\mathrm{a}}$ \\
\hline
\end{tabular}

${ }^{\text {abc }}$ Multiple means comparisons based on Tukey-Kramer Honestly Significant Difference (HSD) test. Levels not connected by the same letter are significantly different. 
ancestor. Thus, we conclude that multiple GS3 haplotypes conferring short seeds occurred independently in the japonica and indica groups. This is in contrast to the mutational history of the long seeded phenotype where a unique, nonsense mutation occurred in the second exon of GS3 in the japonica gene pool or in a japonica-like ancestor and was disseminated into the indica gene pool via introgression during the process of rice domestication (Takano-Kai et al. 2009).

The short or small seeded phenotype of rice was occasionally a target of artificial selection in some restricted areas. The short seeded cultivars JC73-4, JC149, JC157 and JC101 are aromatic cultivars collected from Orissa, India by Oka (1957). Although aromatic rice which is popular as a premium rice in world markets has long seeds and grains, the majority of indigenous Indian aromatic rice varieties have short or medium sized seeds (Singh et al. 2000). Aromatic, short grained rice in India is widely used by local consumers for making kheer (sweet rice) for religious and festive occasions (Rani et al. 2006).

\section{The function of GS3 in rice seed}

GS3 has previously been shown to regulate stigma length and is involved in stigma exsertion in rice. A nonsense mutation in the second exon of GS3 results in an increase in cell number, resulting in stigma elongation (Takano-Kai et al. 2011). In this study, we demonstrated that, in addition to controlling stigma length, GS3 controls cell number in the upper epidermis of the glume. Several genes that regulate cell number have been isolated, and one of them is DENSE AND ERECT PANICLE1 (DEP1), a gene involved in rice grain yield (Huang et al. 2009, Taguchi-Shiobara et al. 2011, Wang et al. 2009, Zhou et al. 2009). DEP1 is reported to carry a previously unknown PEBP-like domain protein sharing some homology with the $\mathrm{N}$ terminus of $G S 3$, and three VWFC domains in the C-terminus (Huang et al. 2009, Wang et al. 2009). The dominant allele at the DEP1 locus (depl) is a gain-of-function mutation that truncates at the Cterminus of DEP1. The effect of depl is to enhance meristematic activity, resulting in reduced length of the inflorescence internode, an increase in the number of grains per panicle, and a consequential increase in grain yield (Huang et al. 2009). The domain structure of depl is very similar to that of the 320-bp, 13-bp, 4-bp and 1 + 3-bp deletion alleles of GS3 identified in this study, because the amino acid sequence of dep 1 lacks two VWFC domains in the C-terminus. Recently, an atypical heterotrimeric G-protein $\gamma$-subunit (AGG3) was identified in Arabidopsis thaliana (Chakravorty et al. 2011). AGG3 contains a $\gamma$-subunit in its $\mathrm{N}$ terminus followed by a putative transmembrane domain and a $\mathrm{C}$-terminal cysteinerich region. The $\gamma$-subunit of AGG3 interacts with the Arabipopsis G-protein $\beta$-subunit (Chakravorty et al. 2011) and regulates organ size and shape in A. thaliana (Li et al. 2012). GS3 and DEP1 in rice are homologues of AGG3 (Chakravorty et al. 2011). Botella (2012) speculates that GS3 and DEP1 are G-protein $\gamma$-subunits and interact with rice G-protein $\beta$-subunit, resulting in the inhibition of seed expansion and enhancement of meristematic activity, respectively. Moreover, Botella (2012) proposes that the cysteine-rich regions (VWFC domain) of GS3 and DEP1 have an inhibitory effect in the $\beta \gamma$ dimer signaling of GS3 and DEP1, therefore the lack of the cysteine-rich region results in increased signaling by $\beta \gamma$ dimer. These interpretations are consistent with the protein structures and phenotypes of the deletion alleles of GS3 identified in this study. Although it remains unclear how GS3 and DEP1 regulate cell number, elucidating that GS3 and DEP1 function as rice heterotrimeric G-proteins may help clarify the regulatory mechanism of cell division and grain development.

\section{Acknowledgments}

This study was partly supported by a grant from the Programme for Promotion of Basic and Applied Researches for innovations in Bio-oriented industry. We are grateful the Biotron Application Center at Kyushu University, for use of the closed greenhouse to grow the transgenic plants.

\section{Literature Cited}

Botella,J.R. (2012) Can heterotrimeric G proteins help to feed the world? Trends Plant Sci. 17: 563-568.

Chakravorty, D., Y.Trusov, W.Zhang, B.R.Acharya, M.B.Sheahan, D.W.McCurdy, S.M.Assmann and J.R.Botella (2011) An atypical heterotrimeric G-protein $\gamma$-subunit is involved in guard cell $\mathrm{K}^{+}$channel regulation and morphological development in Arabidopsis thaliana. Plant J. 67: 840-851.

Dellaporta,S.L., J.Wood and J.B.Hicks (1983) A plant DNA minipreparation: version II. Plant Mol. Biol. Rep. 1: 19-21.

Fan, C, Y.Xing, H.Mao, T.Lu, B.Han, C.Xu, X.Li and Q.Zhang (2006) GS3, a major QTL for grain length and weight and minor QTL for grain width and thickness in rice, encodes a putative transmembrane protein. Theor. Appl. Genet. 112: 1164-1171.

Fraker,E., J.Li and S.McCouch (2004) SSR mapping of Minute, a major gene for grain size, on rice chromosome 3. Rice Genet. Newsl. 21: 21-23.

Huang, X., Q.Qian, Z.Liu, H.Sun, S.He, D.Luo, G.Xia, C.Chu, J.Li and X.Fu (2009) Natural variation at the DEP1 locus enhances grain yield in rice. Nat. Genet. 41: 494-497.

Juliano, B.O. and C.P.Villareal (1993) Grain quality evaluation of world rices. International Rice Research Institute, Manila, Philippines.

Khush,G.S. (1999) Green revolution: preparing for the 21 st century. Genome 42: 646-655.

Li,J., J.Xiao, S.Grandillo, L.Jiang, Y.Wan, Q.Deng, L.Yuan and S.McCouch (2004) QTL detection for rice grain quality traits using an interspecific backcross population derived from cultivated Asian (O. sativa L.) and African (O. glaberrima S) rice. Genome 47: 697-704.

Li,S., Y.Liu, L.Zheng, L.Chen, N.Li, F.Corke, Y.Lu, X.Fu, Z.Zhu, M.W.Bevan et al. (2012) The plant-specific G protein $\gamma$ subunit AGG3 influences organ size and shape in Arabidopsis thaliana. New Phytol. 194: 690-703.

Mao,H., S.Sun, J.Yao, C.Wang, S.Yu, C.Xu, X.Li and Q.Zhang (2010) Linking differential domain function of the GS3 protein to 
natural variation of grain size in rice. Proc. Natl. Acad. Sci. USA 107: 19579-19584.

Mikami,T., M.Takadate, H.Yokoyama, Y.Kawamura, W.Kobayashi, M.Tateyama, K.Maeda, T.Nakahori and Z.Oyamada (2004) A new rice variety 'Tsubuyuki'. Bull. Aomori Agric. Forest. Res. Cent. 40: $39-56$.

Miki,D. and K. Shimamoto (2004) Simple RNAi vectors for stable and transient suppression of gene function in rice. Plant Cell Physiol. 45: 490-495.

Oka,H.I. (1957) Report of study tour to India for collection of rice, 1957. In: Morishima, H. (ed.) Reports of the Study-Tours for Investigation of Wild and Cultivated Rice Species Part F. pp. 1-34.

Rani,N.S., M.K.Pandey, G.S.V.Prasad and I.Sudharshan (2006) Historical significance, grain quality features and precision breeding for improvement of export quality basmati varieties in India. Indian J. Crop Sci. 1: 29-41.

Redoña,E.D. and D.J.Mackill (1998) Quantitative trait locus analysis for rice panicle and grain characteristics. Theor. Appl. Genet. 96: 957-963.

Shomura,A., T.Izawa, K.Ebana, T.Ebitani, H.Kanegae, S.Konishi and M.Yano (2008) Deletion in a gene associated with grain size increased yields during rice domestication. Nat. Genet. 40: 10231028.

Singh,R.K., U.S.Singh and G.S.Khush (2000) Aromatic rices. Oxford \& IBH Pub. Co. Pvt. Ltd., New Delhi, India.

Song,X.J., W.Huang, M.Shi, M.Z.Zhu and H.X.Lin (2007) A QTL for rice grain width and weight encodes a previously unknown RINGtype E3 ubiquitin ligase. Nat. Genet. 39: 623-630.

Taguchi-Shiobara,F., Y.Kawagoe, H.Kato, H.Onodera, A.Tagiri, N.Hara, A.Miyao, H.Hirochika, H.Kitano, M.Yano et al. (2011) A loss-of-function mutation ofrice DENSE PANICLE 1 causes semidwarfness and slightly increased number of spikelets. Breed. Sci. 61: 17-25.

Takamure,I., T.Yamamoto and T.Kinoshita (1991) Determination of loci for $M i$ (Minute grain) and $L k-f$ (Fusayoshi long grain) on the 3rd linkage group in rice. Jpn. J. Breed. 41(Suppl. 1): 322-323.

Takano-Kai, N., H. Jiang, T.Kubo, M. Sweeney, T. Matsumoto, H.
Kanamori, B.Padhukasahasram, C.Bustamante, A.Yoshimura, K. Doi et al. (2009) Evolutionary history of GS3, a gene conferring grain length in rice. Genetics 182: 1323-1334.

Takano-Kai,N., K.Doi and A.Yoshimura (2011) GS3 participates in stigma exsertion as well as seed length in rice. Breed. Sci. 61: 244 250.

Takeda,K. and K. Saito (1977) The inheritance and character expression of the minute gene derived from a rice genetic tester "Minute". Bull. Fac. Agric. Hirosaki Univ. 27: 1-29.

Takeoka, Y. (1976) Histogenesis of lemma in japonica paddy rice. Proc. Crop Sci. Soc. Jpn. 45: 569-581.

Tan, Y.F., Y.Z.Xing, J.X.Li, S.B.Yu, C.G.Xu and Q.F.Zhang (2000) Genetic bases of appearance quality of rice grains in Shanyou 63, an elite rice hybrid. Theor. Appl. Genet. 101: 823-829.

Toki,S. (1997) Rapid and efficient Agrobacterium-mediated transformation in rice. PlantMol. Biol. Rep. 15: 16-21.

Tsunematsu,H., A.Yoshimura, M.Yano and N.Iwata (1995) Quantitative trait locus analysis using recombinant inbred lines and restriction fragment length polymorphism markers in rice. In: Khushu, G.S. (ed.) Rice Genet. Ill, International Rice Research Institute, Manila, Philippines, pp. 619-623.

Unnevehr,L.J., B.Duff and O.Juliano (1992) Consumer demand for rice grain quality. International Rice Research Institute, Manila, Philippines.

Wang, J., T.Nakazaki, S.Chen, W.Chen, H.Saito, T.Tsukiyama, Y. Okumoto, Z.Xu and T.Tanisaka (2009) Identification and characterization of the erect-pose panicle gene $E P$ conferring high grain yield in rice (Oryza sativa L.). Theor. Appl. Genet. 119: 85-91.

Weng,J., S.Gu, X.Wan, H.Gao, T.Guo, N.Su, C.Lei, X.Zhang, Z.Cheng, X.Guo et al. (2008) Isolation and initial characterization of GW5, a major QTL associated with rice grain width and weight. Cell Res. 18: 1199-1209.

Zhou,Y., J.Zhu, Z.Li, C.Yi, J.Liu, H.Zhang, S.Tang, M.Gu and G. Liang (2009) Deletion in a quantitative trait gene qPE9-l associated with panicle erectness improves plant architecture during rice domestication. Genetics 183: 315-324. 